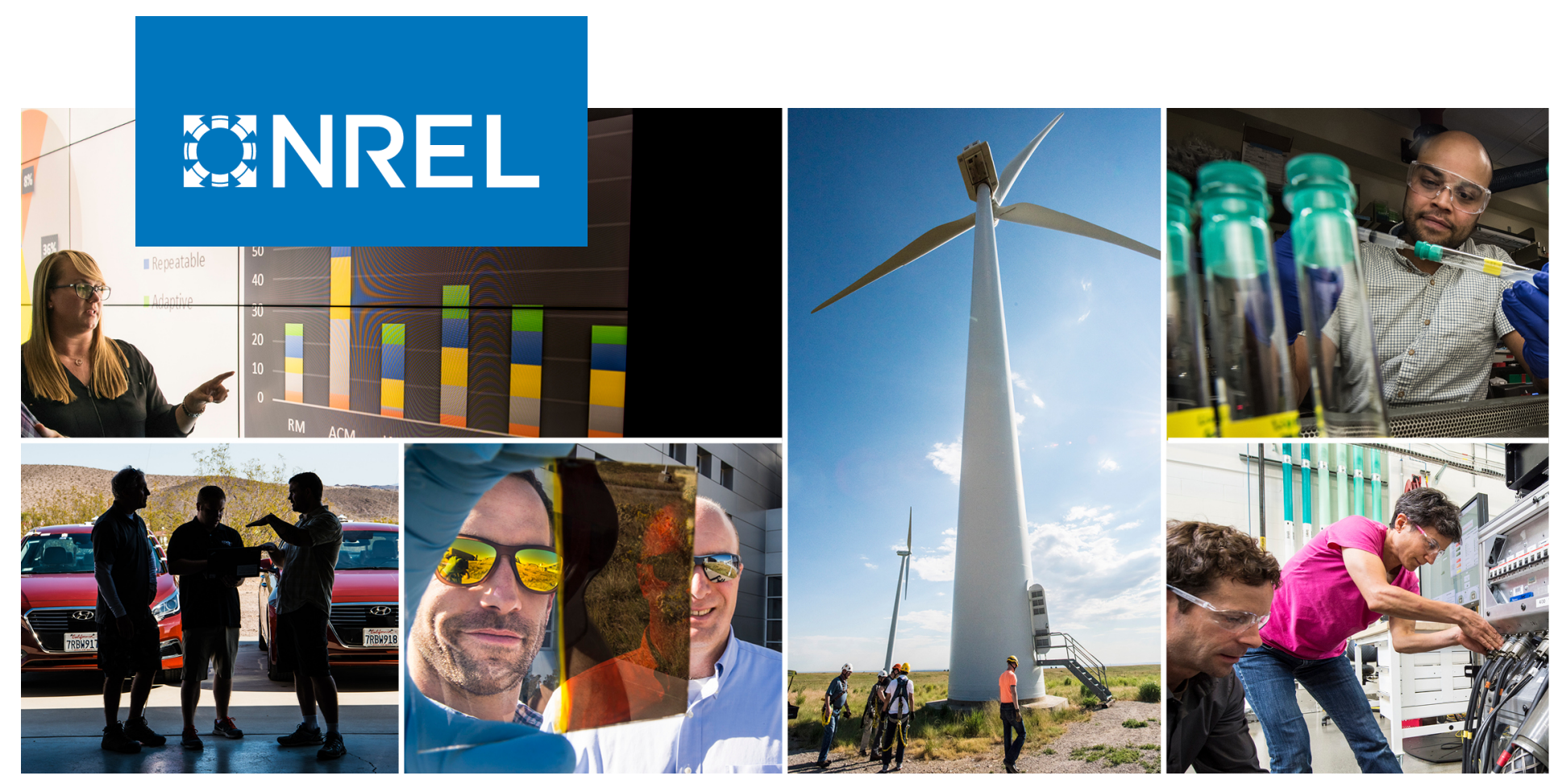

\title{
Summary of Results: Evaluation of Uplight Energy Saving Actions Using ResStock ${ }^{\mathrm{TM}}$
}

Nathan Moore and Dane Christensen

National Renewable Energy Laboratory

Produced under direction of Uplight by the National Renewable Energy

Laboratory (NREL) under Technical Services Agreement number TSA-18-1068.

NREL is a national laboratory of the U.S. Department of Energy Office of Energy Efficiency \& Renewable Energy

Operated by the Alliance for Sustainable Energy, LLC

This report is available at no cost from the National Renewable Energy Laboratory (NREL) at www.nrel.gov/publications.
Strategic Partnership Project Report NREL/TP-5500-76167

August 2020 


\section{GHREL}

\section{Summary of Results: Evaluation of Uplight Energy Saving Actions Using ResStock $^{\mathrm{TM}}$}

\section{Nathan Moore and Dane Christensen}

\section{Suggested Citation}

Moore, Nathan and Dane Christensen. 2020. Summary of Results: Evaluation of Uplight Energy Saving Actions Using ResStock ${ }^{T M}$. Golden, CO: National Renewable Energy Laboratory. NREL/TP-5500-76167. https://www.nrel.gov/docs/fy20osti/76167.pdf.

NREL is a national laboratory of the U.S. Department of Energy Office of Energy Efficiency \& Renewable Energy Operated by the Alliance for Sustainable Energy, LLC

This report is available at no cost from the National Renewable Energy Laboratory (NREL) at www.nrel.gov/publications.

Contract No. DE-AC36-08GO28308
Strategic Partnership Project Report NREL/TP-5500-76167 August 2020

National Renewable Energy Laboratory 15013 Denver West Parkway Golden, CO 80401 303-275-3000 • www.nrel.gov 


\section{NOTICE}

This work was authored by the National Renewable Energy Laboratory, operated by Alliance for Sustainable Energy, LLC, for the U.S. Department of Energy (DOE) under Contract No. DE-AC36-08GO28308. Support for the work was also provided by Uplight under Technical Services Agreement TSA-18-1068. The views expressed in the article do not necessarily represent the views of the DOE or the U.S. Government. The U.S. Government retains and the publisher, by accepting the article for publication, acknowledges that the U.S. Government retains a nonexclusive, paid-up, irrevocable, worldwide license to publish or reproduce the published form of this work, or allow others to do so, for U.S. Government purposes.

This report is available at no cost from the National Renewable Energy Laboratory (NREL) at www.nrel.gov/publications.

U.S. Department of Energy (DOE) reports produced after 1991 and a growing number of pre-1991 documents are available free via www.OSTI.gov.

Cover Photos by Dennis Schroeder: (clockwise, left to right) NREL 51934, NREL 45897, NREL 42160, NREL 45891, NREL 48097, NREL 46526.

NREL prints on paper that contains recycled content. 


\section{Introduction}

As part of a portfolio of product offerings, Uplight produces energy savings estimates to inform a utility's residential customers of the expected benefits that would arise from adopting energy efficiency measures, which are termed "energy saving actions." Uplight's current practice is to use a proprietary building simulation model to estimate energy and cost benefits from these actions, as discussed in Maguire et al. Uplight requested that the National Renewable Energy Laboratory (NREL) provide an independent estimate of the energy savings for a defined list of energy saving actions. NREL performed this study using two established building energy simulation tools: EnergyPlus $^{\circledR}$ (EnergyPlus) and ResStock ${ }^{\mathrm{TM}}$ (Wilson et al.).

A brief summary of the tools, approaches, and methods is provided in this whitepaper's Appendix.

\section{Climate Regions}

Results are summarized by climate region, which are defined as shown in Figure 1.

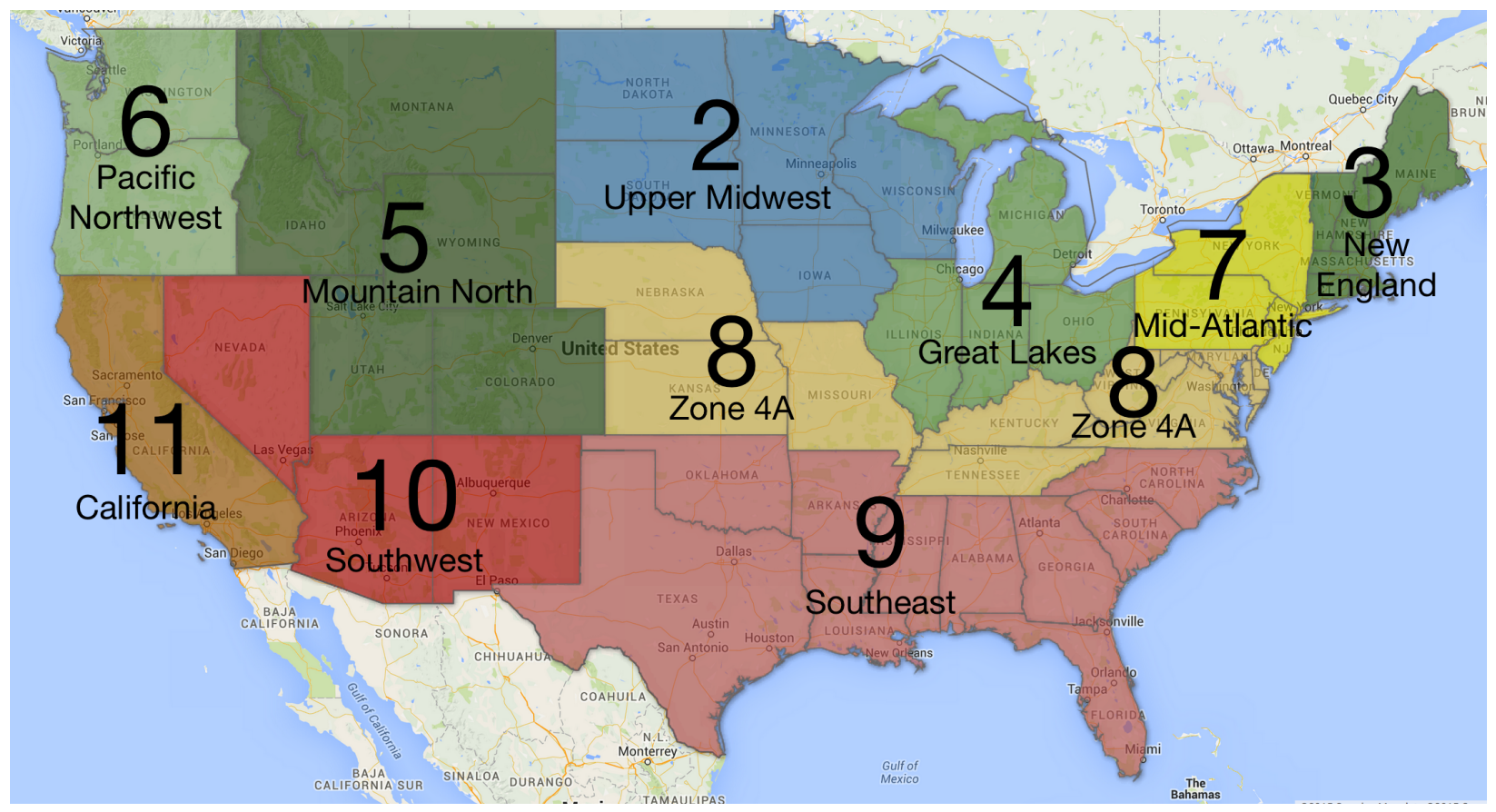

Figure 1. Map defining climate regions

Results are calculated across all ResStock results, based on where the weather station was located within that geographic climate region. Mean and standard deviation of energy savings for each measure were calculated in a weighted fashion (e.g., if a ResStock archetype represented 150 homes, it received five times the weight of an archetype that represented only 30 homes when calculating the mean and standard deviation).

\section{Results by Climate Region}

The following tables summarize the annual mean energy savings for each climate region. 
Table 1. Summary of Annual Household Electricity Savings for Climate Region 2: Upper Midwest

\begin{tabular}{|c|c|c|}
\hline Residential Upgrade & Mean (kWh) & Standard Deviation $(\mathrm{kWh})$ \\
\hline $100 \%$ light-emitting diode lighting & 740 & 696 \\
\hline Air leakage $25 \%$ reduction, with mech. vent. under 7 air changes per hour at 50 pascals & 59 & 515 \\
\hline Baseboard/radiant heating to high efficiency minisplit & $-2,683$ & 9,420 \\
\hline Cease use of clothes dryer & 25 & 48 \\
\hline Central air source heat pump to variable-speed heat pump & 4,024 & 2,035 \\
\hline Close window blinds & 45 & 49 \\
\hline Combustion boiler to condensing boiler & 24 & 59 \\
\hline Combustion furnace to variable-speed heat pump & $-9,539$ & 6,309 \\
\hline Combustion furnace to condensing furnace & 30 & 70 \\
\hline Cook outside in summer & 78 & 43 \\
\hline Cooling setpoint to $80^{\circ} \mathrm{F}$ & 1,062 & 585 \\
\hline Cooling setback from no setback & 735 & 409 \\
\hline Cover pool & 904 & 934 \\
\hline Crawlspace ceiling insulation & 11 & 130 \\
\hline Crawlspace wall insulation & 1,268 & 3,110 \\
\hline Duct sealing and insulation to R- $8,10 \%$ leakage & 181 & 382 \\
\hline Electric furnace to variable-speed heat pump & 9,722 & 4,754 \\
\hline Electric vehicle, 5,000 miles & $-1,852$ & 6 \\
\hline ENERGY STAR clothes dryer & 211 & 187 \\
\hline ENERGY STAR clothes washer & 301 & 243 \\
\hline ENERGY STAR dishwasher & 60 & 59 \\
\hline ENERGY STAR freezer & 150 & 19 \\
\hline ENERGY STAR refrigerator & 206 & 228 \\
\hline ENERGY STAR roof & 66 & 88 \\
\hline ENERGY STAR windows & 443 & 723 \\
\hline Energy recovery ventilation & -394 & 300 \\
\hline Finished basement wall insulation & 211 & 506 \\
\hline Ground source heat pump & $-5,924$ & 6,563 \\
\hline Heat recovery ventilation & -439 & 366 \\
\hline Heat pump clothes dryer & 342 & 199 \\
\hline Heating setbacks from no setbacks & 296 & 690 \\
\hline Install $4 \mathrm{~kW}$ of PV on south-facing roof & 5,294 & 98 \\
\hline Insulate vaulted ceiling & 0 & 6 \\
\hline Insulate water pipes & 45 & 57 \\
\hline Lower heating setpoint by 2 degrees & 215 & 480 \\
\hline Natural ventilation & 78 & 43 \\
\hline $\mathrm{R}-38$ attic insulation & 164 & 463 \\
\hline $\mathrm{R}-49$ attic insulation & 165 & 443 \\
\hline $\mathrm{R}-60$ attic insulation & 142 & 399 \\
\hline Radiant barrier & 46 & 64 \\
\hline Raise cooling setpoint by 2 degrees & 338 & 194 \\
\hline Reduce clothes dryer usage & 159 & 117 \\
\hline Reduce covered pool temp to $78^{\circ} \mathrm{F}$ & 80 & 80 \\
\hline Reduce lighting usage & 95 & 51 \\
\hline Reduce pool pump size & 576 & 198 \\
\hline Reduce uncovered pool temp to $78^{\circ} \mathrm{F}$ & 258 & 267 \\
\hline Reduce water use & 522 & 749 \\
\hline Seasonal energy efficiency ratio 15 air conditioner (AC) & 527 & 345 \\
\hline Seasonal energy efficiency ratio 18 air conditioner (AC) & 666 & 397 \\
\hline Seasonal energy efficiency ratio 24 air conditioner (AC) & 1,139 & 501 \\
\hline Seal rim joist & 593 & 2,255 \\
\hline Spray foam wall insulation & 1,050 & 2,616 \\
\hline Storm windows & 376 & 562 \\
\hline Tank water heater to condensing tank & 50 & 218 \\
\hline Tank water heater to condensing tankless & 1,397 & 1,949 \\
\hline Tank water heater to premium tank & 30 & 200 \\
\hline Tank water heater to tankless & -166 & 679 \\
\hline Unfinished basement wall insulation & 392 & 541 \\
\hline Unplug second refrigerator & 165 & 72 \\
\hline Use low-flow water fixtures & 27 & 59 \\
\hline Wall insulation for uninsulated wood walls & 286 & 1,221 \\
\hline Wash laundry in cold water & 55 & 119 \\
\hline Water heater to 50 -gal heat pump water heater & $-1,221$ & 2,152 \\
\hline Water heater to 80 -gal heat pump water heater & -485 & 1,836 \\
\hline
\end{tabular}


Table 2. Summary of Annual Household Electricity Savings for Climate Region 3: New England

\begin{tabular}{|c|c|c|}
\hline Residential Upgrade & Mean $(\mathrm{kWh})$ & Standard Deviation $(\mathrm{kWh})$ \\
\hline $100 \%$ light-emitting diode lighting & 654 & 610 \\
\hline Air leakage $25 \%$ reduction, with mech. vent. under 7 air changes per hour at 50 pascals & 41 & 259 \\
\hline Baseboard/radiant heating to high efficiency minisplit & $-4,040$ & 5,189 \\
\hline Cease use of clothes dryer & 13 & 32 \\
\hline Close window blinds & 30 & 40 \\
\hline Combustion boiler to condensing boiler & 22 & 44 \\
\hline Combustion furnace to variable-speed heat pump & $-8,243$ & 5,552 \\
\hline Combustion furnace to condensing furnace & 38 & 64 \\
\hline Cook outside in summer & 80 & 42 \\
\hline Cooling setpoint to $80^{\circ} \mathrm{F}$ & 890 & 671 \\
\hline Cooling setback from no setback & 636 & 479 \\
\hline Cover pool & 848 & 878 \\
\hline Crawlspace ceiling insulation & 9 & 87 \\
\hline Crawlspace wall insulation & 274 & 1,021 \\
\hline Duct sealing and insulation to R- $8,10 \%$ leakage & 49 & 101 \\
\hline Electric furnace to variable-speed heat pump & 13,641 & 6,490 \\
\hline Electric vehicle, 5,000 miles & $-1,852$ & 8 \\
\hline ENERGY STAR clothes dryer & 210 & 181 \\
\hline ENERGY STAR clothes washer & 297 & 215 \\
\hline ENERGY STAR dishwasher & 51 & 51 \\
\hline ENERGY STAR freezer & 152 & 16 \\
\hline ENERGY STAR refrigerator & 206 & 224 \\
\hline ENERGY STAR roof & 82 & 147 \\
\hline ENERGY STAR windows & 224 & 356 \\
\hline Energy recovery ventilation & -205 & 215 \\
\hline Finished basement wall insulation & 55 & 245 \\
\hline Ground source heat pump & $-7,632$ & 5,505 \\
\hline Heat recovery ventilation & -215 & 234 \\
\hline Heat pump clothes dryer & 323 & 186 \\
\hline Heating setbacks from no setbacks & 118 & 338 \\
\hline Install $4 \mathrm{~kW}$ of PV on south-facing roof & 5,153 & 78 \\
\hline Insulate vaulted ceiling & 0 & 7 \\
\hline Insulate water pipes & 35 & 50 \\
\hline Lower heating setpoint by 2 degrees & 122 & 332 \\
\hline Natural ventilation & 80 & 42 \\
\hline R-38 attic insulation & 129 & 487 \\
\hline $\mathrm{R}-49$ attic insulation & 118 & 440 \\
\hline R-60 attic insulation & 127 & 447 \\
\hline Radiant barrier & 53 & 132 \\
\hline Raise cooling setpoint by 2 degrees & 311 & 240 \\
\hline Reduce clothes dryer usage & 178 & 106 \\
\hline Reduce covered pool temp to $78^{\circ} \mathrm{F}$ & 72 & 75 \\
\hline Reduce lighting usage & 84 & 45 \\
\hline Reduce pool pump size & 550 & 181 \\
\hline Reduce uncovered pool temp to $78^{\circ} \mathrm{F}$ & 242 & 251 \\
\hline Reduce water use & 387 & 662 \\
\hline Seasonal energy efficiency ratio 15 air conditioner (AC) & 469 & 292 \\
\hline Seasonal energy efficiency ratio 18 air conditioner (AC) & 570 & 329 \\
\hline Seasonal energy efficiency ratio 24 air conditioner (AC) & 1,013 & 413 \\
\hline Seal rim joist & 12 & 774 \\
\hline Spray foam wall insulation & 279 & 974 \\
\hline Storm windows & 180 & 257 \\
\hline Tank water heater to condensing tank & 16 & 158 \\
\hline Tank water heater to condensing tankless & 1,186 & 1,802 \\
\hline Tank water heater to premium tank & 4 & 125 \\
\hline Tank water heater to tankless & -240 & 664 \\
\hline Unfinished basement wall insulation & 151 & 189 \\
\hline Unplug second refrigerator & 167 & 72 \\
\hline Use low-flow water fixtures & 23 & 51 \\
\hline Wall insulation for uninsulated wood walls & 182 & 706 \\
\hline Wash laundry in cold water & 38 & 93 \\
\hline Water heater to 50 -gal heat pump water heater & $-1,459$ & 1,993 \\
\hline Water heater to 80 -gal heat pump water heater & -897 & 1,728 \\
\hline
\end{tabular}


Table 3. Summary of Annual Household Electricity Savings for Climate Region 4: Great Lakes

\begin{tabular}{|c|c|c|}
\hline Residential Upgrade & Mean $(\mathrm{kWh})$ & Standard Deviation $(\mathrm{kWh})$ \\
\hline $100 \%$ light-emitting diode lighting & 660 & 633 \\
\hline Air leakage $25 \%$ reduction, with mech. vent. under 7 air changes per hour at 50 pascals & 123 & 498 \\
\hline Baseboard/radiant heating to high efficiency minisplit & 3,773 & 11,441 \\
\hline Cease use of clothes dryer & 24 & 45 \\
\hline Central air source heat pump to variable-speed heat pump & 4,389 & 2,431 \\
\hline Close window blinds & 45 & 48 \\
\hline Combustion boiler to condensing boiler & 22 & 53 \\
\hline Combustion furnace to variable-speed heat pump & $-7,632$ & 5,436 \\
\hline Combustion furnace to condensing furnace & 22 & 56 \\
\hline Cook outside in summer & 66 & 45 \\
\hline Cooling setpoint to $80^{\circ} \mathrm{F}$ & 1,226 & 672 \\
\hline Cooling setback from no setback & 835 & 464 \\
\hline Cover pool & 851 & 885 \\
\hline Crawlspace ceiling insulation & 17 & 264 \\
\hline Crawlspace wall insulation & 1,069 & 2,832 \\
\hline Duct sealing and insulation to R- $8,10 \%$ leakage & 224 & 455 \\
\hline Electric furnace to variable-speed heat pump & 10,842 & 5,651 \\
\hline Electric vehicle, 5,000 miles & $-1,852$ & 13 \\
\hline ENERGY STAR clothes dryer & 204 & 180 \\
\hline ENERGY STAR clothes washer & 245 & 226 \\
\hline ENERGY STAR dishwasher & 53 & 53 \\
\hline ENERGY STAR freezer & 151 & 23 \\
\hline ENERGY STAR refrigerator & 201 & 221 \\
\hline ENERGY STAR roof & 97 & 160 \\
\hline ENERGY STAR windows & 429 & 650 \\
\hline Energy recovery ventilation & -310 & 288 \\
\hline Finished basement wall insulation & 189 & 443 \\
\hline Ground source heat pump & $-5,496$ & 7,070 \\
\hline Heat recovery ventilation & -351 & 345 \\
\hline Heat pump clothes dryer & 336 & 193 \\
\hline Heating setbacks from no setbacks & 238 & 523 \\
\hline Install $4 \mathrm{~kW}$ of PV on south-facing roof & 5,044 & 130 \\
\hline Insulate vaulted ceiling & 0 & 13 \\
\hline Insulate water pipes & 35 & 48 \\
\hline Lower heating setpoint by 2 degrees & 296 & 605 \\
\hline Natural ventilation & 66 & 45 \\
\hline $\mathrm{R}-38$ attic insulation & 276 & 800 \\
\hline $\mathrm{R}-49$ attic insulation & 246 & 724 \\
\hline R-60 attic insulation & 255 & 721 \\
\hline Radiant barrier & 82 & 180 \\
\hline Raise cooling setpoint by 2 degrees & 402 & 229 \\
\hline Reduce clothes dryer usage & 132 & 115 \\
\hline Reduce covered pool temp to $78^{\circ} \mathrm{F}$ & 75 & 77 \\
\hline Reduce lighting usage & 85 & 49 \\
\hline Reduce pool pump size & 550 & 187 \\
\hline Reduce uncovered pool temp to $78^{\circ} \mathrm{F}$ & 243 & 253 \\
\hline Reduce water use & 414 & 667 \\
\hline Seasonal energy efficiency ratio 15 air conditioner (AC) & 597 & 388 \\
\hline Seasonal energy efficiency ratio 18 air conditioner (AC) & 706 & 433 \\
\hline Seasonal energy efficiency ratio 24 air conditioner (AC) & 1,283 & 552 \\
\hline Seal rim joist & 479 & 2,176 \\
\hline Spray foam wall insulation & 912 & 2,247 \\
\hline Storm windows & 368 & 491 \\
\hline Tank water heater to condensing tank & 49 & 166 \\
\hline Tank water heater to condensing tankless & 1,009 & 1,638 \\
\hline Tank water heater to premium tank & 23 & 158 \\
\hline Tank water heater to tankless & -96 & 533 \\
\hline Unfinished basement wall insulation & 362 & 475 \\
\hline Unplug second refrigerator & 166 & 76 \\
\hline Use low-flow water fixtures & 23 & 53 \\
\hline Wall insulation for uninsulated wood walls & 584 & 1,636 \\
\hline Wash laundry in cold water & 40 & 98 \\
\hline Water heater to 50 -gal heat pump water heater & $-1,241$ & 1,892 \\
\hline Water heater to 80 -gal heat pump water heater & -604 & 1,528 \\
\hline
\end{tabular}


Table 4. Summary of Annual Household Electricity Savings for Climate Region 5: Mountain North

\begin{tabular}{|c|c|c|}
\hline Residential Upgrade & Mean (kWh) & Standard Deviation $(\mathrm{kWh})$ \\
\hline $100 \%$ light-emitting diode lighting & 646 & 615 \\
\hline Air leakage $25 \%$ reduction, with mech. vent. under 7 air changes per hour at 50 pascals & -25 & 348 \\
\hline Baseboard/radiant heating to high efficiency minisplit & 848 & 6,495 \\
\hline Cease use of clothes dryer & 12 & 31 \\
\hline Central air source heat pump to variable-speed heat pump & 3,840 & 1,905 \\
\hline Close window blinds & 30 & 47 \\
\hline Combustion boiler to condensing boiler & 10 & 51 \\
\hline Combustion furnace to variable-speed heat pump & $-4,632$ & 3,871 \\
\hline Combustion furnace to condensing furnace & 22 & 72 \\
\hline Cook outside in summer & 83 & 41 \\
\hline Cooling setpoint to $80^{\circ} \mathrm{F}$ & 769 & 693 \\
\hline Cooling setback from no setback & 558 & 501 \\
\hline Cover pool & 867 & 870 \\
\hline Crawlspace ceiling insulation & 9 & 178 \\
\hline Crawlspace wall insulation & 628 & 1,770 \\
\hline Duct sealing and insulation to R- $8,10 \%$ leakage & 148 & 304 \\
\hline Electric furnace to variable-speed heat pump & 6,583 & 3,959 \\
\hline Electric vehicle, 5,000 miles & $-1,852$ & 8 \\
\hline ENERGY STAR clothes dryer & 200 & 176 \\
\hline ENERGY STAR clothes washer & 288 & 201 \\
\hline ENERGY STAR dishwasher & 48 & 47 \\
\hline ENERGY STAR freezer & 147 & 18 \\
\hline ENERGY STAR refrigerator & 194 & 212 \\
\hline ENERGY STAR roof & 56 & 147 \\
\hline ENERGY STAR windows & 285 & 473 \\
\hline Energy recovery ventilation & -365 & 253 \\
\hline Finished basement wall insulation & 127 & 315 \\
\hline Ground source heat pump & $-4,464$ & 4,931 \\
\hline Heat recovery ventilation & -390 & 303 \\
\hline Heat pump clothes dryer & 320 & 182 \\
\hline Heating setbacks from no setbacks & 213 & 478 \\
\hline Install $4 \mathrm{~kW}$ of PV on south-facing roof & 5,992 & 270 \\
\hline Insulate vaulted ceiling & -0 & 7 \\
\hline Insulate water pipes & 25 & 45 \\
\hline Lower heating setpoint by 2 degrees & 214 & 455 \\
\hline Natural ventilation & 83 & 41 \\
\hline $\mathrm{R}-38$ attic insulation & 163 & 430 \\
\hline $\mathrm{R}-49$ attic insulation & 147 & 394 \\
\hline R-60 attic insulation & 163 & 413 \\
\hline Radiant barrier & 48 & 92 \\
\hline Raise cooling setpoint by 2 degrees & 253 & 233 \\
\hline Reduce clothes dryer usage & 174 & 104 \\
\hline Reduce covered pool temp to $78^{\circ} \mathrm{F}$ & 73 & 76 \\
\hline Reduce lighting usage & 82 & 47 \\
\hline Reduce pool pump size & 549 & 182 \\
\hline Reduce uncovered pool temp to $78^{\circ} \mathrm{F}$ & 247 & 249 \\
\hline Reduce water use & 330 & 611 \\
\hline Seasonal energy efficiency ratio 15 air conditioner (AC) & 467 & 317 \\
\hline Seasonal energy efficiency ratio 18 air conditioner (AC) & 640 & 383 \\
\hline Seasonal energy efficiency ratio 24 air conditioner (AC) & 941 & 442 \\
\hline Seal rim joist & 316 & 1,376 \\
\hline Spray foam wall insulation & 522 & 1,063 \\
\hline Storm windows & 256 & 384 \\
\hline Tank water heater to condensing tank & 9 & 143 \\
\hline Tank water heater to condensing tankless & 863 & 1,616 \\
\hline Tank water heater to premium tank & -5 & 143 \\
\hline Tank water heater to tankless & -123 & 503 \\
\hline Unfinished basement wall insulation & 245 & 359 \\
\hline Unplug second refrigerator & 159 & 70 \\
\hline Use low-flow water fixtures & 13 & 49 \\
\hline Wall insulation for uninsulated wood walls & 244 & 668 \\
\hline Wash laundry in cold water & 35 & 93 \\
\hline Water heater to 50 -gal heat pump water heater & $-1,514$ & 1,881 \\
\hline Water heater to 80 -gal heat pump water heater & -918 & 1,549 \\
\hline
\end{tabular}


Table 5. Summary of Annual Household Electricity Savings for Climate Region 6: Pacific Northwest

\begin{tabular}{|c|c|c|}
\hline Residential Upgrade & Mean (kWh) & Standard Deviation (kWh) \\
\hline $100 \%$ light-emitting diode lighting & 472 & 470 \\
\hline Air leakage $25 \%$ reduction, with mech. vent. under 7 air changes per hour at 50 pascals & 238 & 537 \\
\hline Baseboard/radiant heating to high efficiency minisplit & 9,724 & 8,164 \\
\hline Cease use of clothes dryer & 34 & 57 \\
\hline Central air source heat pump to variable-speed heat pump & 2,743 & 1,850 \\
\hline Close window blinds & 16 & 37 \\
\hline Combustion boiler to condensing boiler & 13 & 41 \\
\hline Combustion furnace to variable-speed heat pump & $-3,240$ & 3,103 \\
\hline Combustion furnace to condensing furnace & 16 & 41 \\
\hline Cook outside in summer & 83 & 42 \\
\hline Cooling setpoint to $80^{\circ} \mathrm{F}$ & 531 & 562 \\
\hline Cooling setback from no setback & 390 & 413 \\
\hline Cover pool & 773 & 822 \\
\hline Crawlspace ceiling insulation & 184 & 792 \\
\hline Crawlspace wall insulation & 1,387 & 2,411 \\
\hline Duct sealing and insulation to R- $8,10 \%$ leakage & 386 & 757 \\
\hline Electric furnace to variable-speed heat pump & 7,071 & 5,138 \\
\hline Electric vehicle, 5,000 miles & $-1,852$ & 23 \\
\hline ENERGY STAR clothes dryer & 193 & 169 \\
\hline ENERGY STAR clothes washer & 373 & 224 \\
\hline ENERGY STAR dishwasher & 73 & 67 \\
\hline ENERGY STAR freezer & 135 & 34 \\
\hline ENERGY STAR refrigerator & 164 & 196 \\
\hline ENERGY STAR roof & -22 & 216 \\
\hline ENERGY STAR windows & 353 & 524 \\
\hline Energy recovery ventilation & -247 & 238 \\
\hline Finished basement wall insulation & 244 & 412 \\
\hline Ground source heat pump & $-1,409$ & 6,233 \\
\hline Heat recovery ventilation & -285 & 297 \\
\hline Heat pump clothes dryer & 328 & 179 \\
\hline Heating setbacks from no setbacks & 491 & 834 \\
\hline Install $4 \mathrm{~kW}$ of PV on south-facing roof & 4,736 & 348 \\
\hline Insulate vaulted ceiling & -0 & 21 \\
\hline Insulate water pipes & 49 & 56 \\
\hline Lower heating setpoint by 2 degrees & 636 & 967 \\
\hline Natural ventilation & 83 & 42 \\
\hline $\mathrm{R}-38$ attic insulation & 375 & 1,164 \\
\hline $\mathrm{R}-49$ attic insulation & 298 & 952 \\
\hline R-60 attic insulation & 320 & 943 \\
\hline Radiant barrier & 60 & 233 \\
\hline Raise cooling setpoint by 2 degrees & 190 & 203 \\
\hline Reduce clothes dryer usage & 175 & 95 \\
\hline Reduce covered pool temp to $78^{\circ} \mathrm{F}$ & 71 & 74 \\
\hline Reduce lighting usage & 60 & 44 \\
\hline Reduce pool pump size & 511 & 172 \\
\hline Reduce uncovered pool temp to $78^{\circ} \mathrm{F}$ & 221 & 236 \\
\hline Reduce water use & 804 & 703 \\
\hline Seasonal energy efficiency ratio 15 air conditioner (AC) & 302 & 223 \\
\hline Seasonal energy efficiency ratio 18 air conditioner (AC) & 382 & 262 \\
\hline Seasonal energy efficiency ratio 24 air conditioner (AC) & 627 & 334 \\
\hline Seal rim joist & 978 & 1,902 \\
\hline Spray foam wall insulation & 1,858 & 2,885 \\
\hline Storm windows & 360 & 461 \\
\hline Tank water heater to condensing tank & 25 & 294 \\
\hline Tank water heater to condensing tankless & 1,963 & 1,823 \\
\hline Tank water heater to premium tank & 27 & 209 \\
\hline Tank water heater to tankless & -334 & 697 \\
\hline Unfinished basement wall insulation & 279 & 390 \\
\hline Unplug second refrigerator & 143 & 83 \\
\hline Use low-flow water fixtures & 41 & 69 \\
\hline Wall insulation for uninsulated wood walls & 1,068 & 2,138 \\
\hline Wash laundry in cold water & 86 & 125 \\
\hline Water heater to 50 -gal heat pump water heater & -328 & 1,701 \\
\hline Water heater to 80 -gal heat pump water heater & 73 & 1,508 \\
\hline
\end{tabular}


Table 6. Summary of Annual Household Electricity Savings for Climate Region 7: Mid-Atlantic

\begin{tabular}{|c|c|c|}
\hline Residential Upgrade & Mean (kWh) & Standard Deviation $(\mathrm{kWh})$ \\
\hline $100 \%$ light-emitting diode lighting & 679 & 638 \\
\hline Air leakage $25 \%$ reduction, with mech. vent. under 7 air changes per hour at 50 pascals & 162 & 461 \\
\hline Baseboard/radiant heating to high efficiency minisplit & $-1,276$ & 8,501 \\
\hline Cease use of clothes dryer & 18 & 40 \\
\hline Central air source heat pump to variable-speed heat pump & 4,205 & 2,376 \\
\hline Close window blinds & 46 & 51 \\
\hline Combustion boiler to condensing boiler & 23 & 52 \\
\hline Combustion furnace to variable-speed heat pump & $-7,276$ & 5,589 \\
\hline Combustion furnace to condensing furnace & 26 & 62 \\
\hline Cook outside in summer & 63 & 46 \\
\hline Cooling setpoint to $80^{\circ} \mathrm{F}$ & 1,312 & 746 \\
\hline Cooling setback from no setback & 906 & 520 \\
\hline Cover pool & 889 & 900 \\
\hline Crawlspace ceiling insulation & 7 & 191 \\
\hline Crawlspace wall insulation & 337 & 1,045 \\
\hline Duct sealing and insulation to R- $8,10 \%$ leakage & 131 & 250 \\
\hline Electric furnace to variable-speed heat pump & 12,608 & 6,335 \\
\hline Electric vehicle, 5,000 miles & $-1,852$ & 14 \\
\hline ENERGY STAR clothes dryer & 207 & 181 \\
\hline ENERGY STAR clothes washer & 230 & 215 \\
\hline ENERGY STAR dishwasher & 50 & 50 \\
\hline ENERGY STAR freezer & 153 & 22 \\
\hline ENERGY STAR refrigerator & 205 & 223 \\
\hline ENERGY STAR roof & 113 & 181 \\
\hline ENERGY STAR windows & 380 & 548 \\
\hline Energy recovery ventilation & -179 & 245 \\
\hline Finished basement wall insulation & 169 & 381 \\
\hline Ground source heat pump & $-6,765$ & 6,759 \\
\hline Heat recovery ventilation & -201 & 285 \\
\hline Heat pump clothes dryer & 331 & 190 \\
\hline Heating setbacks from no setbacks & 184 & 444 \\
\hline Install $4 \mathrm{~kW}$ of PV on south-facing roof & 5,064 & 120 \\
\hline Insulate vaulted ceiling & 0 & 14 \\
\hline Insulate water pipes & 33 & 46 \\
\hline Lower heating setpoint by 2 degrees & 249 & 558 \\
\hline Natural ventilation & 63 & 46 \\
\hline $\mathrm{R}-38$ attic insulation & 224 & 646 \\
\hline R-49 attic insulation & 210 & 594 \\
\hline R-60 attic insulation & 228 & 604 \\
\hline Radiant barrier & 83 & 170 \\
\hline Raise cooling setpoint by 2 degrees & 440 & 258 \\
\hline Reduce clothes dryer usage & 133 & 117 \\
\hline Reduce covered pool temp to $78^{\circ} \mathrm{F}$ & 72 & 77 \\
\hline Reduce lighting usage & 87 & 48 \\
\hline Reduce pool pump size & 554 & 188 \\
\hline Reduce uncovered pool temp to $78^{\circ} \mathrm{F}$ & 254 & 257 \\
\hline Reduce water use & 367 & 616 \\
\hline Seasonal energy efficiency ratio 15 air conditioner (AC) & 652 & 430 \\
\hline Seasonal energy efficiency ratio 18 air conditioner (AC) & 762 & 474 \\
\hline Seasonal energy efficiency ratio 24 air conditioner (AC) & 1,389 & 612 \\
\hline Seal rim joist & -76 & 830 \\
\hline Spray foam wall insulation & 584 & 1,645 \\
\hline Storm windows & 309 & 371 \\
\hline Tank water heater to condensing tank & 52 & 145 \\
\hline Tank water heater to condensing tankless & 996 & 1,597 \\
\hline Tank water heater to premium tank & 25 & 152 \\
\hline Tank water heater to tankless & -99 & 530 \\
\hline Unfinished basement wall insulation & 269 & 313 \\
\hline Unplug second refrigerator & 167 & 75 \\
\hline Use low-flow water fixtures & 21 & 49 \\
\hline Wall insulation for uninsulated wood walls & 395 & 1,225 \\
\hline Wash laundry in cold water & 35 & 89 \\
\hline Water heater to 50 -gal heat pump water heater & $-1,290$ & 1,834 \\
\hline Water heater to 80 -gal heat pump water heater & -701 & 1,477 \\
\hline
\end{tabular}


Table 7. Summary of Annual Household Electricity Savings for Climate Region 8: Zone 4A

\begin{tabular}{|c|c|c|}
\hline Residential Upgrade & Mean $(\mathrm{kWh})$ & Standard Deviation $(\mathrm{kWh})$ \\
\hline $100 \%$ light-emitting diode lighting & 659 & 637 \\
\hline Air leakage $25 \%$ reduction, with mech. vent. under 7 air changes per hour at 50 pascals & 263 & 630 \\
\hline Baseboard/radiant heating to high efficiency minisplit & 7,580 & 8,569 \\
\hline Cease use of clothes dryer & 42 & 57 \\
\hline Central air source heat pump to variable-speed heat pump & 4,119 & 2,358 \\
\hline Close window blinds & 72 & 69 \\
\hline Combustion boiler to condensing boiler & 36 & 69 \\
\hline Combustion furnace to variable-speed heat pump & $-2,908$ & 2,885 \\
\hline Combustion furnace to condensing furnace & 31 & 73 \\
\hline Cook outside in summer & 89 & 40 \\
\hline Cooling setpoint to $80^{\circ} \mathrm{F}$ & 1,639 & 743 \\
\hline Cooling setback from no setback & 1,052 & 489 \\
\hline Cover pool & 870 & 894 \\
\hline Crawlspace ceiling insulation & 23 & 282 \\
\hline Crawlspace wall insulation & 1,702 & 2,812 \\
\hline Duct sealing and insulation to R- $8,10 \%$ leakage & 496 & 678 \\
\hline Electric furnace to variable-speed heat pump & 10,265 & 5,554 \\
\hline Electric vehicle, 5,000 miles & $-1,852$ & 18 \\
\hline ENERGY STAR clothes dryer & 209 & 184 \\
\hline ENERGY STAR clothes washer & 355 & 224 \\
\hline ENERGY STAR dishwasher & 69 & 61 \\
\hline ENERGY STAR freezer & 149 & 24 \\
\hline ENERGY STAR refrigerator & 200 & 222 \\
\hline ENERGY STAR roof & 131 & 197 \\
\hline ENERGY STAR windows & 687 & 762 \\
\hline Energy recovery ventilation & -345 & 338 \\
\hline Finished basement wall insulation & 300 & 360 \\
\hline Ground source heat pump & $-1,804$ & 6,138 \\
\hline Heat recovery ventilation & -418 & 421 \\
\hline Heat pump clothes dryer & 355 & 199 \\
\hline Heating setbacks from no setbacks & 457 & 661 \\
\hline Install $4 \mathrm{~kW}$ of PV on south-facing roof & 5,425 & 112 \\
\hline Insulate vaulted ceiling & -0 & 18 \\
\hline Insulate water pipes & 57 & 53 \\
\hline Lower heating setpoint by 2 degrees & 549 & 773 \\
\hline Natural ventilation & 89 & 40 \\
\hline $\mathrm{R}-38$ attic insulation & 372 & 931 \\
\hline $\mathrm{R}-49$ attic insulation & 365 & 854 \\
\hline R-60 attic insulation & 404 & 866 \\
\hline Radiant barrier & 122 & 243 \\
\hline Raise cooling setpoint by 2 degrees & 561 & 264 \\
\hline Reduce clothes dryer usage & 187 & 109 \\
\hline Reduce covered pool temp to $78^{\circ} \mathrm{F}$ & 74 & 78 \\
\hline Reduce lighting usage & 84 & 52 \\
\hline Reduce pool pump size & 554 & 191 \\
\hline Reduce uncovered pool temp to $78^{\circ} \mathrm{F}$ & 248 & 256 \\
\hline Reduce water use & 702 & 687 \\
\hline Seasonal energy efficiency ratio 15 air conditioner (AC) & 965 & 640 \\
\hline Seasonal energy efficiency ratio 18 air conditioner (AC) & 1,047 & 684 \\
\hline Seasonal energy efficiency ratio 24 air conditioner (AC) & 1,988 & 883 \\
\hline Seal rim joist & 831 & 2,203 \\
\hline Spray foam wall insulation & 1,701 & 2,534 \\
\hline Storm windows & 551 & 496 \\
\hline Tank water heater to condensing tank & 131 & 310 \\
\hline Tank water heater to condensing tankless & 1,759 & 1,702 \\
\hline Tank water heater to premium tank & 82 & 236 \\
\hline Tank water heater to tankless & -143 & 615 \\
\hline Unfinished basement wall insulation & 550 & 493 \\
\hline Unplug second refrigerator & 162 & 75 \\
\hline Use low-flow water fixtures & 36 & 64 \\
\hline Wall insulation for uninsulated wood walls & 922 & 1,846 \\
\hline Wash laundry in cold water & 63 & 103 \\
\hline Water heater to 50 -gal heat pump water heater & -275 & 1,669 \\
\hline Water heater to 80 -gal heat pump water heater & 186 & 1,451 \\
\hline
\end{tabular}


Table 8. Summary of Annual Household Electricity Savings for Climate Region 9: Southeast

\begin{tabular}{|c|c|c|}
\hline Residential Upgrade & Mean (kWh) & Standard Deviation $(\mathrm{kWh})$ \\
\hline $100 \%$ light-emitting diode lighting & 618 & 581 \\
\hline Air leakage $25 \%$ reduction, with mech. vent. under 7 air changes per hour at 50 pascals & 288 & 469 \\
\hline Baseboard/radiant heating to high efficiency minisplit & 7,499 & 4,098 \\
\hline Cease use of clothes dryer & 49 & 58 \\
\hline Central air source heat pump to variable-speed heat pump & 3,195 & 1,725 \\
\hline Close window blinds & 111 & 98 \\
\hline Combustion boiler to condensing boiler & 25 & 61 \\
\hline Combustion furnace to variable-speed heat pump & 856 & 1,793 \\
\hline Combustion furnace to condensing furnace & 28 & 81 \\
\hline Cook outside in summer & 86 & 41 \\
\hline Cooling setpoint to $80^{\circ} \mathrm{F}$ & 2,023 & 864 \\
\hline Cooling setback from no setback & 1,167 & 530 \\
\hline Cover pool & 826 & 843 \\
\hline Crawlspace ceiling insulation & 1 & 130 \\
\hline Crawlspace wall insulation & 1,184 & 2,010 \\
\hline Duct sealing and insulation to R-8, $10 \%$ leakage & 516 & 492 \\
\hline Electric furnace to variable-speed heat pump & 6,139 & 3,454 \\
\hline Electric vehicle, 5,000 miles & $-1,852$ & 16 \\
\hline ENERGY STAR clothes dryer & 209 & 185 \\
\hline ENERGY STAR clothes washer & 321 & 202 \\
\hline ENERGY STAR dishwasher & 68 & 55 \\
\hline ENERGY STAR freezer & 157 & 26 \\
\hline ENERGY STAR refrigerator & 224 & 246 \\
\hline ENERGY STAR roof & 342 & 323 \\
\hline ENERGY STAR windows & 851 & 748 \\
\hline Energy recovery ventilation & -249 & 299 \\
\hline Finished basement wall insulation & 309 & 216 \\
\hline Ground source heat pump & $-1,749$ & 3,615 \\
\hline Heat recovery ventilation & -317 & 381 \\
\hline Heat pump clothes dryer & 362 & 201 \\
\hline Heating setbacks from no setbacks & 464 & 642 \\
\hline Install $4 \mathrm{~kW}$ of PV on south-facing roof & 5,647 & 206 \\
\hline Insulate vaulted ceiling & 0 & 16 \\
\hline Insulate water pipes & 68 & 45 \\
\hline Lower heating setpoint by 2 degrees & 425 & 559 \\
\hline Natural ventilation & 86 & 41 \\
\hline R-38 attic insulation & 402 & 938 \\
\hline $\mathrm{R}-49$ attic insulation & 441 & 926 \\
\hline $\mathrm{R}-60$ attic insulation & 485 & 940 \\
\hline Radiant barrier & 232 & 378 \\
\hline Raise cooling setpoint by 2 degrees & 815 & 352 \\
\hline Reduce clothes dryer usage & 183 & 111 \\
\hline Reduce covered pool temp to $78^{\circ} \mathrm{F}$ & 71 & 74 \\
\hline Reduce lighting usage & 79 & 46 \\
\hline Reduce pool pump size & 525 & 177 \\
\hline Reduce uncovered pool temp to $78^{\circ} \mathrm{F}$ & 236 & 241 \\
\hline Reduce water use & 666 & 571 \\
\hline Seasonal energy efficiency ratio 15 air conditioner (AC) & 1,585 & 1,048 \\
\hline Seasonal energy efficiency ratio 18 air conditioner (AC) & 1,325 & 1,076 \\
\hline Seasonal energy efficiency ratio 24 air conditioner (AC) & 3,155 & 1,403 \\
\hline Seal rim joist & 395 & 1,574 \\
\hline Spray foam wall insulation & 1,123 & 1,427 \\
\hline Storm windows & 535 & 353 \\
\hline Tank water heater to condensing tank & 175 & 271 \\
\hline Tank water heater to condensing tankless & 1,573 & 1,363 \\
\hline Tank water heater to premium tank & 111 & 190 \\
\hline Tank water heater to tankless & -26 & 456 \\
\hline Unfinished basement wall insulation & 546 & 337 \\
\hline Unplug second refrigerator & 173 & 79 \\
\hline Use low-flow water fixtures & 29 & 60 \\
\hline Wall insulation for uninsulated wood walls & 487 & 953 \\
\hline Wash laundry in cold water & 45 & 70 \\
\hline Water heater to 50 -gal heat pump water heater & 144 & 1,279 \\
\hline Water heater to 80 -gal heat pump water heater & 423 & 1,179 \\
\hline
\end{tabular}


Table 9. Summary of Annual Household Electricity Savings for Climate Region 10: Southwest

\begin{tabular}{|c|c|c|}
\hline Residential Upgrade & Mean $(\mathrm{kWh})$ & Standard Deviation $(\mathrm{kWh})$ \\
\hline $100 \%$ light-emitting diode lighting & 609 & 567 \\
\hline Air leakage $25 \%$ reduction, with mech. vent. under 7 air changes per hour at 50 pascals & 162 & 340 \\
\hline Baseboard/radiant heating to high efficiency minisplit & 5,567 & 5,397 \\
\hline Cease use of clothes dryer & 25 & 38 \\
\hline Central air source heat pump to variable-speed heat pump & 2,869 & 1,803 \\
\hline Close window blinds & 99 & 121 \\
\hline Combustion boiler to condensing boiler & 3 & 18 \\
\hline Combustion furnace to variable-speed heat pump & 38 & 3,409 \\
\hline Combustion furnace to condensing furnace & 9 & 41 \\
\hline Cook outside in summer & 70 & 44 \\
\hline Cooling setpoint to $80^{\circ} \mathrm{F}$ & 1,233 & 7,53 \\
\hline Cooling setback from no setback & 861 & 538 \\
\hline Cover pool & 828 & 830 \\
\hline Crawlspace ceiling insulation & 3 & 77 \\
\hline Crawlspace wall insulation & 940 & 1,674 \\
\hline Duct sealing and insulation to R-8, $10 \%$ leakage & 548 & 539 \\
\hline Electric furnace to variable-speed heat pump & 3,675 & 4,956 \\
\hline Electric vehicle, 5,000 miles & $-1,852$ & 16 \\
\hline ENERGY STAR clothes dryer & 204 & 178 \\
\hline ENERGY STAR clothes washer & 249 & 194 \\
\hline ENERGY STAR dishwasher & 51 & 47 \\
\hline ENERGY STAR freezer & 156 & 25 \\
\hline ENERGY STAR refrigerator & 222 & 247 \\
\hline ENERGY STAR roof & 419 & 429 \\
\hline ENERGY STAR windows & 725 & 797 \\
\hline Energy recovery ventilation & -226 & 199 \\
\hline Finished basement wall insulation & 319 & 249 \\
\hline Ground source heat pump & $-3,138$ & 4,018 \\
\hline Heat recovery ventilation & -251 & 224 \\
\hline Heat pump clothes dryer & 334 & 187 \\
\hline Heating setbacks from no setbacks & 218 & 507 \\
\hline Install $4 \mathrm{~kW}$ of PV on south-facing roof & 6,800 & 89 \\
\hline Insulate vaulted ceiling & 0 & 16 \\
\hline Insulate water pipes & 47 & 47 \\
\hline Lower heating setpoint by 2 degrees & 229 & 443 \\
\hline Natural ventilation & 70 & 44 \\
\hline $\mathrm{R}-38$ attic insulation & 320 & 788 \\
\hline $\mathrm{R}-49$ attic insulation & 328 & 744 \\
\hline R-60 attic insulation & 369 & 758 \\
\hline Radiant barrier & 203 & 310 \\
\hline Raise cooling setpoint by 2 degrees & 517 & 332 \\
\hline Reduce clothes dryer usage & 153 & 111 \\
\hline Reduce covered pool temp to $78^{\circ} \mathrm{F}$ & 68 & 74 \\
\hline Reduce lighting usage & 78 & 44 \\
\hline Reduce pool pump size & 519 & 173 \\
\hline Reduce uncovered pool temp to $78^{\circ} \mathrm{F}$ & 237 & 237 \\
\hline Reduce water use & 390 & 529 \\
\hline Seasonal energy efficiency ratio 15 air conditioner (AC) & 1,715 & 1,306 \\
\hline Seasonal energy efficiency ratio 18 air conditioner (AC) & 1,405 & 1,291 \\
\hline Seasonal energy efficiency ratio 24 air conditioner (AC) & 2,938 & 1,687 \\
\hline Seal rim joist & 369 & 1,307 \\
\hline Spray foam wall insulation & 1,112 & 1,466 \\
\hline Storm windows & 499 & 400 \\
\hline Tank water heater to condensing tank & 88 & 202 \\
\hline Tank water heater to condensing tankless & 919 & 1,286 \\
\hline Tank water heater to premium tank & 65 & 175 \\
\hline Tank water heater to tankless & -9 & 378 \\
\hline Unfinished basement wall insulation & 671 & 419 \\
\hline Unplug second refrigerator & 171 & 78 \\
\hline Use low-flow water fixtures & 16 & 49 \\
\hline Wall insulation for uninsulated wood walls & 375 & 869 \\
\hline Wash laundry in cold water & 24 & 62 \\
\hline Water heater to 50 -gal heat pump water heater & -545 & 1,357 \\
\hline Water heater to 80 -gal heat pump water heater & -274 & 1,179 \\
\hline
\end{tabular}


Table 10. Summary of Annual Household Electricity Savings for Climate Region 11: California

\begin{tabular}{|c|c|c|}
\hline Residential Upgrade & Mean (kWh) & Standard Deviation $(\mathrm{kWh})$ \\
\hline $100 \%$ light-emitting diode lighting & 580 & 558 \\
\hline Air leakage $25 \%$ reduction, with mech. vent. under 7 air changes per hour at 50 pascals & 7 & 217 \\
\hline Baseboard/radiant heating to high efficiency minisplit & 3,905 & 3,470 \\
\hline Cease use of clothes dryer & 3 & 25 \\
\hline Central air source heat pump to variable-speed heat pump & 2,164 & 1,626 \\
\hline Close window blinds & 44 & 71 \\
\hline Combustion boiler to condensing boiler & 0 & 27 \\
\hline Combustion furnace to variable-speed heat pump & -196 & 1,930 \\
\hline Combustion furnace to condensing furnace & 1 & 22 \\
\hline Cook outside in summer & 48 & 42 \\
\hline Cooling setpoint to $80^{\circ} \mathrm{F}$ & 1,275 & 1,176 \\
\hline Cooling setback from no setback & 916 & 845 \\
\hline Cover pool & 800 & 831 \\
\hline Crawlspace ceiling insulation & -14 & 115 \\
\hline Crawlspace wall insulation & 236 & 1,061 \\
\hline Duct sealing and insulation to R-8, $10 \%$ leakage & 211 & 329 \\
\hline Electric furnace to variable-speed heat pump & 3,677 & 3,407 \\
\hline Electric vehicle, 5,000 miles & $-1,852$ & 16 \\
\hline ENERGY STAR clothes dryer & 198 & 177 \\
\hline ENERGY STAR clothes washer & 141 & 159 \\
\hline ENERGY STAR dishwasher & 36 & 35 \\
\hline ENERGY STAR freezer & 152 & 26 \\
\hline ENERGY STAR refrigerator & 210 & 234 \\
\hline ENERGY STAR roof & 225 & 375 \\
\hline ENERGY STAR windows & 355 & 500 \\
\hline Energy recovery ventilation & -202 & 203 \\
\hline Finished basement wall insulation & 104 & 140 \\
\hline Ground source heat pump & $-2,966$ & 2,894 \\
\hline Heat recovery ventilation & -211 & 221 \\
\hline Heat pump clothes dryer & 314 & 180 \\
\hline Heating setbacks from no setbacks & 212 & 547 \\
\hline Install $4 \mathrm{~kW}$ of PV on south-facing roof & 6,260 & 274 \\
\hline Insulate vaulted ceiling & 0 & 16 \\
\hline Insulate water pipes & 18 & 35 \\
\hline Lower heating setpoint by 2 degrees & 212 & 496 \\
\hline Natural ventilation & 48 & 42 \\
\hline R-38 attic insulation & 199 & 661 \\
\hline $\mathrm{R}-49$ attic insulation & 210 & 651 \\
\hline $\mathrm{R}-60$ attic insulation & 228 & 664 \\
\hline Radiant barrier & 138 & 298 \\
\hline Raise cooling setpoint by 2 degrees & 482 & 433 \\
\hline Reduce clothes dryer usage & 90 & 104 \\
\hline Reduce covered pool temp to $78^{\circ} \mathrm{F}$ & 68 & 73 \\
\hline Reduce lighting usage & 74 & 46 \\
\hline Reduce pool pump size & 516 & 174 \\
\hline Reduce uncovered pool temp to $78^{\circ} \mathrm{F}$ & 229 & 238 \\
\hline Reduce water use & 122 & 335 \\
\hline Seasonal energy efficiency ratio 15 air conditioner (AC) & 895 & 779 \\
\hline Seasonal energy efficiency ratio 18 air conditioner (AC) & 838 & 799 \\
\hline Seasonal energy efficiency ratio 24 air conditioner (AC) & 1,855 & 1,156 \\
\hline Seal rim joist & 11 & 974 \\
\hline Spray foam wall insulation & 239 & 923 \\
\hline Storm windows & 240 & 280 \\
\hline Tank water heater to condensing tank & 3 & 149 \\
\hline Tank water heater to condensing tankless & 224 & 826 \\
\hline Tank water heater to premium tank & -19 & 158 \\
\hline Tank water heater to tankless & -24 & 235 \\
\hline Unfinished basement wall insulation & 172 & 222 \\
\hline Unplug second refrigerator & 169 & 79 \\
\hline Use low-flow water fixtures & 7 & 30 \\
\hline Wall insulation for uninsulated wood walls & 139 & 639 \\
\hline Wash laundry in cold water & 8 & 42 \\
\hline Water heater to 50-gal heat pump water heater & $-1,405$ & 1,311 \\
\hline Water heater to 80 -gal heat pump water heater & $-1,009$ & 960 \\
\hline
\end{tabular}




\section{Bibliography}

EnergyPlus. U.S. Department of Energy Building Technologies Office, managed by NREL, 2019. https://energyplus. net/.

Maguire, J., S. Horowitz, N. Moore, and P. Sullivan. Validation of Tendril TrueHome Using Software-to-Software Comparison. Tech. rep. NREL/TP-5500-70116. National Renewable Energy Laboratory, 2017. https://www.nrel. gov/docs/fy18osti/70116.pdf.

OpenStudio. Developed in collaboration by NREL, ANL, LBNL, ORNL, and PNNL, 2019. https://openstudio.net/.

QGIS Project, 2020. https://www.qgis.org/en/site/.

Wilson, E., C. Christensen, S. Horowitz, J. Robertson, and J. Maguire. Energy Efficiency Potential in the U.S. SingleFamily Housing Stock. Tech.rep. NREL/TP-5500-68670. NREL, 2017. https://www.nrel.gov/docs/fy180sti/68670. pdf. Also see https://resstock.nrel.gov/. 


\title{
Appendix. Review of Methods and Tools
}

\author{
Methodology \\ Approach \\ NREL used ResStock (Wilson et al.) to simulate approximately 300,000 homes across the contiguous United States \\ in order to evaluate how savings vary across important building features such as size, climate, and vintage. Res- \\ Stock's multiplier for each archetypal home modeled allowed the 300,000 homes to be scaled up such that the study \\ represents the approximately 100 million homes in the United States.
}

Each of these homes was first simulated in the baseline configuration, and then again with each appropriate energy saving action applied to it. The difference in annual energy consumption for each action was then calculated to determine energy saved.

\section{Energy Saving Actions}

Uplight provided NREL with a list of energy saving actions, with appropriate detail on how such actions are implemented in Uplight's existing software workflow. NREL's first task was to establish which actions could be implemented in the tools chosen for this independent analysis. Not all energy saving actions could be simulated, because for some actions there was either no applicable technology or human behavior model in EnergyPlus, we lacked input data to confidently and accurately represent the action in EnergyPlus, or both.

After reviewing all energy saving actions, 63 were identified as appropriate for this independent study. NREL then developed a method for mapping these actions into the ResStock workflow for comparative analysis.

\section{Tools Used}

\section{EnergyPlus}

EnergyPlus is the U.S. Department of Energy (DOE)'s premiere building energy simulation tool. An open-source multicontributor effort led by NREL, EnergyPlus is an established software for whole-building energy simulation used by architects, engineers, and researchers. Its primary purpose is for estimating energy consumption in buildings and providing design guidance by comparing alternative solutions. It is used as the simulation engine within OpenStudio ${ }^{\circledR}$, and it is behind the scenes of some of the world's leading building design software platforms.

\section{ResStock}

NREL recently developed a simulation package for performing analysis on the nation's entire residential building stock. The ResStock tool (Wilson et al.) uses a best-in-class database of housing stock information to perform largescale energy analysis, leveraging DOE's open-source building energy modeling tools, EnergyPlus (EnergyPlus) and OpenStudio (OpenStudio).

NREL and Uplight agreed that ResStock was appropriate for this effort, based on the following benefits:

- It uses large public and private data sources to produce an open-source input database that is peer reviewed and publicly accessible

- It statistically samples to increase accuracy in representing the diversity of real-world buildings

- It uses detailed subhourly simulations of the homes

- It relies on high-performance computing to increase the number of buildings, thereby producing highly granular results.

\section{Results}

ResStock outputs a set of files showing specific characteristics of the home as modeled, energy use per sector of the home (lighting, cooling, water heating, heating, miscellaneous electric loads (MELs), etc.) and total gross and net energy use of the home. Net energy use can differ from total energy use in the case of on-site energy generation, 
typically photovoltaic (PV) panels. NREL gathered the annual energy output (total and net) for electricity and natural gas for each home before and after each upgrade. Electricity savings, in kilowatt-hour (kWh), were calculated by subtracting the base home's energy use from the upgraded home's energy use.

\section{Outcomes}

These annual energy savings data were compiled in a database, which can be queried to examine how energy usage is affected by vintage, square footage, climate region, fuel type, and a number of other factors.

\section{Inspection of Results}

The data were inspected in several ways to evaluate the quality of results, and to identify and correct any simulation errors that may have occurred. Many blank spaces were found in the data, which were traced to nonsensical inputs, as expected. For instance, an upgrade that improves a combustion heater (furnace/boiler) to a condensing version of the same would not be applied to homes with electric baseboard heat. All records where savings in both gas and electricity were "null" were removed. This reduced the total number of records by roughly one-third. Further investigation showed the results from simulations of dehumidifiers and window air conditioner (AC) were not realistic. While the exact cause for this is under investigation, all dehumidifier and window $\mathrm{AC}$ results in this analysis were eliminated.

NREL also developed visualization methods to examine the results and view trends. Savings values were aggregated by weather station to observe how energy savings are affected by climate. QGIS (QGIS Project) was used to map the results, producing colored points across the country that represent average savings for each upgrade. One map showing an example of mean savings for a given measure across the nation is shown in Figure 2. 


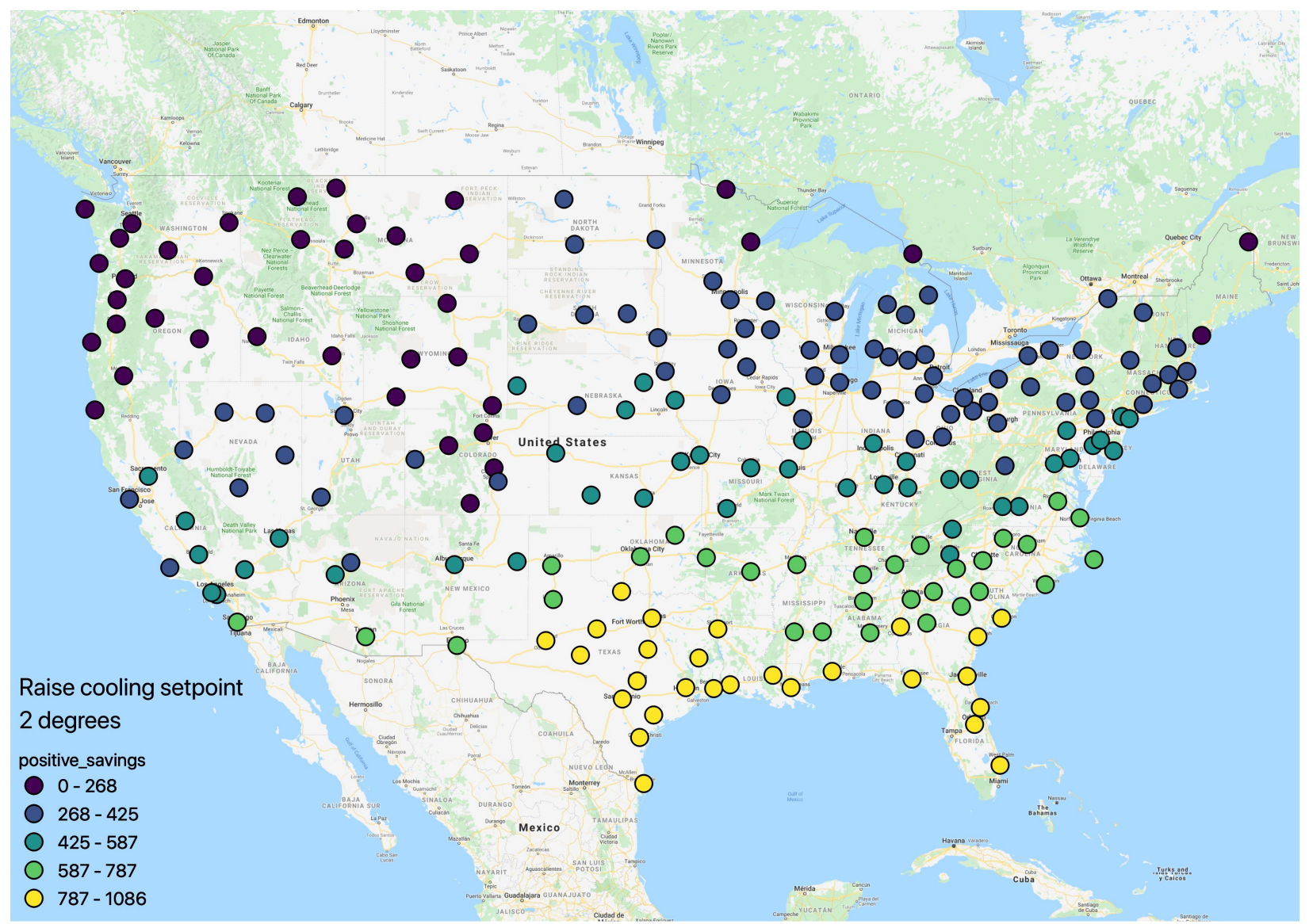

Figure 2. Map of annual mean household electric energy savings from raising cooling setpoint 2 degrees, in $\mathrm{kWh}$
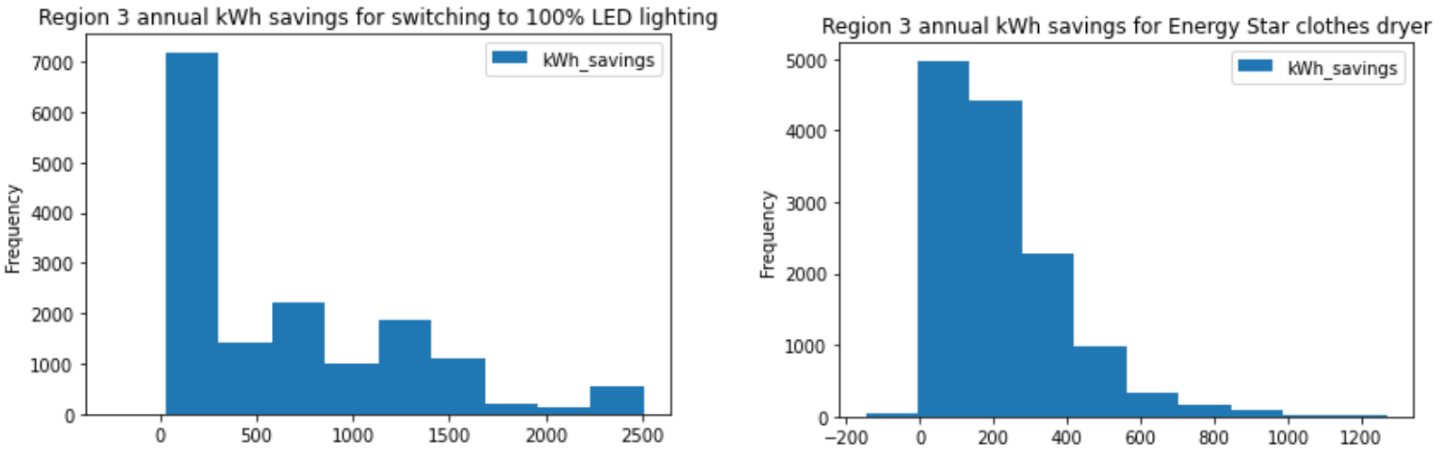

Figure 3. Histogram of annual household energy savings for LED lighting (left) and ENERGY STAR clothes dryer (right) in Region 3 homes

This report is available at no cost from the National Renewable Energy Laboratory at www.nrel.gov/publications. 